\title{
Investigating the influence of different thermodynamic paths on the structural relaxation in a glass forming polymer melt
}

\author{
Christoph Bennemann \\ Wolfgang Paul \\ Jörg Baschnagel \\ Kurt Binder \\ Institut für Physik \\ 55099 Mainz \\ Germany
}

August 13, 2018

\begin{abstract}
We present results from Molecular Dynamics simulations of the thermal glass transition in a dense polymer melt. In previous work we compared the simulation data with the idealized version of mode coupling theory (MCT) and found that the theory provides a good description of the dynamics above the critical temperature. In order to investigate the influence of different thermodynamic paths on the structural relaxation ( $\alpha$-process), we performed simulations for three different pressures and are thus able to give a sketch of the critical line of MCT in the pressuretemperature-plane $[(p, T)$-plane $]$, where, according to the idealised version of MCT, an ergodic-nonergodic transition should occur. Furthermore, by cooling our system along two different paths (an isobar and an isochor), with the same impact point on the critical line, we demonstrate that neither the critical temperature nor the exponent $\gamma$ depend on the chosen path.
\end{abstract}

PACS: 61.20.Ja,64.70.Pf,61.25.Hq,83.10.Nn

submitted to J. Phys.: Condens. Matter

\section{Introduction}

The understanding of the glass transition has been a longstanding problem of condensed matter physics and materials science [1, 2, 3, 4. The term glass transition is used to describe a phenomenon, where a solidification of the liquid without simultaneous crystallisation occurs. In a narrow temperature region the viscosity of the material increases by some 14 orders of magnitude, and, although the typical time scales of the system 
change tremendously, no significant change in the structure is observed and the material remains amorphous.

Experimental research in this area of scientific interest has been conducted for more than a hundred years [1, 5]. Since then a large number of phenomenological theories were proposed, such as the free volume theory [6, 7, 8] or the Gibbs-Di-Marzio theory [9, 10, 11, [2], which tried to explain the characteristic features of the glass transition. These theories developed models, mainly based on physical intuition, from which thermodynamic properties and the temperature dependence of the viscosity, i.e., the Vogel-Fulcher law [1], could be derived. However, an explicit relationship between the model parameters and the microscopic properties of the glass former remained hard to establish, and a detailed description of the shape of dynamic correlation functions was not attempted.

In recent years the mode coupling theory (MCT) was successful in describing a broad range of features observed in experiments 13, 14, 15, 16, 17 and simulations 18, 19, 20, 21, 22, 23, 24, 25, 26] (also see [27] for an overview) in a temperature region close to a critical temperature $T_{c}$, which in general lies above the empirically defined glass transition temperature $T_{g}$. The critical temperature is associated with a change in dynamics from a liquid-like to a solid-like behaviour without any concomitant modification of the glass former's structure. In contrast to most earlier theories this theory starts from well known microscopic dynamics and uses techniques already applied in the field of critical dynamics to derive a set of dynamic equations for the density correlation functions of the system [28, 29, 30, 31] (also see [32, 33] for review articles). It made novel predictions on the temporal behaviour of the density correlations, which could be tested experimentally and generated new research in this area.

The ideal version of the theory states that at the critical temperature the glass former should show a transition from ergodicity to nonergodicity [29]. However, in real systems this transition is in general absent (an exception being (probably) some colloidal glasses [14]), because additional relaxation processes, which are not included in ideal MCT, assure that the system is able to relax even below the ideal transition temperature. In contrast, the extended version of MCT [29, 30, 31] does account for these additional processes in terms of activated hopping processes and therefore predicts that the transition is indeed avoided. Since sufficiently above the critical temperature the contribution of jump processes to the dynamics of the glass former is negligible, the ideal version of MCT often describes experimental data in this temperature regime very well. Only if one is sufficiently close to or even below the critical temperature, one has to apply extended MCT [13, 19. However, such temperatures are seldomly accessible in computer simulations of polymer systems, at least if equilibrated melts are required. Thus, in the following section we will concentrate on the predictions of ideal MCT.

Although MCT has been applied to experimental data numerous times, many aspects of the theory still remain to be thoroughly investigated. Little is known on the influence of external thermodynamic parameters on the transition temperature (although see [34, 35, 36, 37) or the $\alpha$-process. Since in general higher pressure causes higher densities, which in turn means that the movement of an individual particle is more hindered, an increase in pressure results in an increase of the transition temperature. This effect is well known for the glass transition temperature [4. Hence, it is possible to cause a glass transition by sole increase of the pressure, which indeed has been observed in experiment [37]. In general the thermodynamic parameter space (for instance, the $(p, T)$-plane) should decompose 
into two areas, a fluid phase and an ideal glass phase, both separated by a critical line at which the transition to nonergodicity should occur. Since MCT applies to systems in thermodynamic equilibrium, the position of the critical line should be independent on the thermodynamic path chosen upon cooling. Furthermore, close to the critical line, the exponents of the theory, which determine most of the quantitative behaviour of the glass former, depend solely on the impact point of the thermodynamic path on the critical line, granted one does not choose a too exotic path, e.g. one that runs almost parallel to the critical line. Therefore two different thermodynamic paths, which have the same impact point on the critical line, should not only yield the same critical temperature, but the quantitative behaviour of the systems, described in terms of MCT, should be the same along both of them. To our knowledge, so far this prediction has never been verified.

Thus, we decided to conduct a study of the influence of pressure on the parameters of ideal MCT, which also should enable us to give a sketch of the critical line in the $(p, T)$-plane. To this end, we chose a model for a polymer melt, which has already been used in an earlier study of the glass transition [26]. Clearly, because of the connectivity of the monomers along the chain, our model is by no means a simple liquid, and therefore certainly not the kind of system ideal MCT was originally developed for. On the other hand, polymers, according to Angell's classification scheme [38, mostly belong to the fragile glass formers, to which MCT has been applied successfully, and are extremely good glass formers, i.e. "supercooled" melts in thermal equilibrium can be prepared very well. In previous work [26] it was demonstrated that it is possible to equilibrate our model well in the regime of the supercooled melt, a prerequisite to apply MCT, and that the $\alpha$ relaxation behaviour is compatible with MCT. Since we were able to simulate the system in the isochoric (NVT) as well as in the isobaric (NpT) ensemble, we try to test the prediction of thermodynamic path independence in the present paper. To this end, we cooled our system along an isochoric path, which shared the impact point on the critical line with one of the isobaric paths. As in our earlier work 26], we concentrate on the $\alpha$-relaxation behaviour in this study. An analysis of the $\beta$-relaxation can be found in Ref. [39].

The remainder of the paper is organised as follows. In section II we briefly describe our model and the simulation technique. By performing simulations along different isobars we were able to investigate the influence of pressure on the critical temperature. The results of these simulations will be discussed in section III. In section IV we will perform a test of the thermodynamic path independence, and in section $\mathrm{V}$ conclusions will be drawn.

\section{Model and Simulation Technique}

For modelling the inter- and intramolecular forces we used a bead-spring model derived from the one suggested by Kremer and Grest 40] and also used in several recent simulations [41, 42]. However, we included here also the attractive part of the Lennard-Jones potential, since previous work on a lattice model for a glassy polymer melt 43, 44, had shown that without such an attraction the model would produce a negative thermal expansion coefficient. The model of Kremer et al. 40, 41, 42] is close to an athermal model of polymer melts and hence does not exhibit a glass transition driven by temperature at all. 
As in our past simulations each chain consisted of 10 beads with mass $m$ set to unity. Although these chains are rather short, they already show the static behaviour characteristic of long polymers in the melt (e.g. Gaussian statistics for the end-to-end distance distribution, a Debeye scattering law for the single chain structure factor, etc.). Note that each bond in this model would correspond to $n \approx 3-6$ covalent bonds along the backbone of a real chain, if one were to map this coarse-grained model onto a real polymer. Between all monomers there acted a truncated Lennard-Jones potential:

$$
U_{\mathrm{LJ}}\left(r_{i j}\right)=\left\{\begin{array}{rl}
4 \epsilon\left[\left(\frac{\sigma}{r_{i j}}\right)^{12}-\left(\frac{\sigma}{r_{i j}}\right)^{6}\right]+C & : \quad r_{i j}<2 \cdot 2^{\frac{1}{6}} \sigma \\
0 & : \quad r_{i j} \geq 2 \cdot 2^{\frac{1}{6}} \sigma
\end{array},\right.
$$

where $C$ was a constant which guaranteed that the potential was continuous everywhere. Since it was not our aim to simulate a specific polymer, we used Lennard-Jones units, where $\epsilon$ and $\sigma$ are set to unity. Note that this means that all quantities are dimensionless. In addition to the Lennard-Jones potential a FENE backbone potential was applied along the chain:

$$
U_{\mathrm{F}}\left(r_{i j}\right)=-\frac{k}{2} R_{0}^{2} \ln \left[1-\left(\frac{r_{i j}}{R_{0}}\right)^{2}\right] .
$$

The parameters of the potential were taken as $k=30$ and $R_{0}=1.5$, guaranteeing a certain stiffness of the bonds while avoiding high frequency modes and chain crossing. Furthermore, with these parameters we set the favoured bond length to a value slightly smaller than the length favoured by the Lennard-Jones potential. Thus we introduced two different incompatible length scales in our system, which prevents the emergence of long range order (i.e. crystal formation) at lower temperatures.

Unlike previous lattice models for the thermally driven glass transition of polymers [43, 44], the present model has a qualitatively reasonable equation of state with a positive thermal expansion coefficient, and can easily be studied under constant density or constant pressure. It allows to study motion and structure from local scales (motions in the neighbour cage) upto large scales.

In order to keep the temperature fixed, all simulations were performed using a NoséHoover thermostat [45, 46, 47]. In this technique the model system is coupled to a heat bath, which represents an additional degree of freedom. To set the system to a desired pressure, the size of the simulation box was adjusted to yield the correct density at each temperature. The resulting configurations were used as start configurations for runs in the canonical ensemble, where the size of the simulation box was kept fixed. Only during these canonical runs dynamic correlation functions for the further analysis were calculated. A more thorough discussion of the simulation technique applied can be found elsewhere [26, 48]. Here we only emphasize that we have carefully checked that the Nosé-Hoover thermostat does not lead to any artefacts in the dynamics of the single chain correlators and local properties that were studied here [48]. Note also that our chain length $N=10$ was short enough that our results are not affected at all by chain entanglement effects.

Altogether we performed simulations at more than 40 different points in the thermodynamic phase space. At each point 10 independent configurations were simulated, each consisting of 120 polymer chains of 10 monomers. With this we were able to perform 
simulations along three isobars and one isochor. Table 1 shows which temperatures were simulated in which ensemble.

In order to equilibrate an individual system at lower temperatures one had to simulate for very long times ( $>10^{6} \mathrm{MD}$-steps). Generally, the equilibration of the lowest temperature in a given ensemble lasted as long as the sum of all equilibration times at higher temperatures of the same ensemble. Altogether the simulations consumed an equivalent of approximately $10 \mathrm{CPU}$-years on a PentiumPro processor run at $180 \mathrm{Mhz}$.

\section{Dynamical properties at different pressures}

As already discussed in the opening paragraph the glass transition manifests itself by a steep increase of the relaxation times by several orders of magnitude. In order to extract these time scales from the simulation data we computed a number of dynamical quantities, like the incoherent intermediate dynamic structure factor:

$$
\phi_{q}^{\mathrm{s}}(t)=\left\langle\frac{1}{N} \sum_{i=1}^{N} \mathrm{e}^{\mathrm{i} \mathbf{q} \cdot\left(\mathbf{r}_{i}(t)-\mathbf{r}_{i}(0)\right)}\right\rangle
$$

where $N$ stands for the total number of monomers in the melt. This function measures the self correlation of the particle positions at different times, and, by varying the wave-vector q, at different length scales.

Recently, orientational degrees of freedom and their relaxational behaviour have come into focus of theoretical research on the glass transition [49, 50]. Results of molecular dynamic simulations for a fluid consisting of diatomic molecules [22, 23, 24] illustrated that there can be significant differences between orientational and translational relaxation. Such differences are also observed in experiments (see Ref. [55], for instance). Clearly, it should be interesting to check, whether we could find any differences between orientational and translational relaxation in our model. Hence, we also calculated the orientational correlation of the end-to-end vector:

$$
E_{n}(t) \equiv\left\langle L_{n}\left(\frac{\mathbf{e}(t) \cdot \mathbf{e}(0)}{\|\mathbf{e}(t)\|\|\mathbf{e}(0)\|}\right)\right\rangle, n=1,2, \ldots
$$

where $L_{n}$ stands for the $n$th Legendre-polynomial, $\mathbf{e}(t)$ is the end-to-end vector of a polymer at time $t$, and $\|\mathbf{e}\|$ is the length of the end-to-end vector at time $t$. The same formula can be applied to measure the dynamical correlation of a bond vector $\mathbf{b}(t)$ :

$$
B_{n}(t) \equiv\left\langle L_{n}\left(\frac{\mathbf{b}(t) \cdot \mathbf{b}(0)}{\|\mathbf{b}(t)\|\|\mathbf{b}(0)\|}\right)\right\rangle, n=1,2, \ldots
$$

Equations (44) and (5) characterize the reorientation dynamics of the largest and of the smallest vectors along the backbone of a chain. In the analysis, we only calculated the first and second polynomial, since these quantities can be measured by dielectric relaxation and light scattering, respectively.

With these three dynamical correlation functions we define the following correlation times:

$$
\phi_{q}^{\mathrm{s}}\left(\tau_{q}\right)=0.3 \quad E_{n}\left(\tau_{E_{n}}\right)=0.3 \quad B_{n}\left(\tau_{B_{n}}\right)=0.3
$$


We have computed a number of other related quantities as well, such as the Rouse-modes of the system or the mean-square-displacements, which are discussed in other publications [26, 39, 56.

\subsection{Behaviour of dynamical correlators}

As shown in Fig. 11 and Fig. 2, the correlators decay in one step at high temperatures, while at lower temperatures a two step process starts to emerge, which becomes the more pronounced, the lower the temperature. The emergence of a plateau in the decay is related to the cage effect [29], where an individual monomer is trapped by its surrounding particles. The average time a monomer needs to escape from the cage of its neighbours increases with decreasing temperature, which explains the extension of the plateau. The presence of a two step relaxation, the so-called $\beta$-relaxation (onto and off of the plateau) and $\alpha$-relaxation (off of the plateau and long-time structural relaxation), is a common feature of glass formers, and is also predicted by MCT.

As can be seen from the plots, the qualitative behaviour is not affected by the applied pressure, although at higher pressure the two step process starts to show up at higher temperatures already. Furthermore, while the height of the plateau depends on the specific correlator, it hardly varies with pressure. The orientational correlators [first and second Legendre-polynomial, see Eqs. (4) and (5)] exhibit a rather high plateau value which is often close to unity (the plateau of the first Legendre-polynomial is always larger than that of the second). Therefore the two step process is only visible on magnification. Clearly, the contribution of the $\alpha$-process to the overall relaxation of a correlator depends on the quantity considered.

Another characteristic of glass forming liquids is that close to the critical temperature the time temperature superposition principle should hold for the $\alpha$-relaxation. One therefore has to rescale a dynamical correlator by a suitably defined $\alpha$-relaxation time and to check whether the curves fall on a master curve in the $\alpha$-regime. As we reported in our earlier work for $p=1$ (and constant volume) [26], this is indeed the case. Here, we additionally observe that our data also obey a time-temperature-pressure superposition principle, i.e., in the $\alpha$-regime data taken from different isobars collapse on a single master curve. This is illustrated for a number of different dynamic correlators in Fig. 3., where fourteen different curves are included in one plot. Similar behaviour has been observed in experiments on orthoterphenyl [36], but, to the best of our knowledge, this is the first report from computer simulations for such a behavior.

\subsection{Behaviour of relaxation times}

Figure 1 and 2 show that on lowering the temperature an increase of the relaxation times by several orders of magnitude takes place, as expected for a glass forming liquid. By means of asymptotic expansions the idealised MCT derives a number of predictions concerning the behaviour of the liquid close to the critical temperature. One finds that sufficiently close to $T_{c}$ the increase of the $\alpha$-relaxation times can be described by the following formula:

$$
\tau=\tau^{0}\left(T-T_{c}\right)^{-\gamma}
$$


where $\tau^{0}$ is an amplitude which depends on the specific relaxation time considered, and $\gamma$ is a parameter of the theory which should be the same for all correlation times, if the corresponding correlator couples to density fluctuations. Furthermore our analysis of the $\beta$-regime suggested that $\gamma$ should take the value of $\gamma=2.09$ for the isobar $p=1.0$ [39].

At all pressures investigated, it is indeed possible to locate a temperature interval, where the increase of the relaxation times can be described by Eq. (7). When applying Eq. (7), $\tau^{0}, T_{c}$, and $\gamma$ were treated as adjustable parameters. Although it is not incompatible with MCT that rotational and translational degrees of freedom freeze at different state points in the temperature-density plane, as was demonstrated in recent publications 49, 50, our analysis suggests that it is possible to find a critical temperature for all isobaric paths, which is independent of the specific correlator and solely a function of the pressure considered. The critical temperatures and densities we obtained are listed in Table 2. Note that the error for the critical temperature for $p=1.0$ is smaller than for the other pressures because a larger number of temperatures was simulated for this isobar. The pressure dependence of the critical temperature is depicted in Fig. 田. As expected, the critical temperature increases with increasing pressure as also calculated for LennardJones models in [34. As one can also see from Table 2 within the error bars the quantity $\rho_{c} T_{c}^{-1 / 4}$ is a constant at the mode coupling critical point as also found experimentally in e.g. [37 and in the simulation of soft sphere models [51, 52, 53 and for Lennard-Jones mixtures [54]. The value we found is within the error bars identical to the Lennard-Jones value in [54].

Figure 5 shows a double logarithmic plot of $\alpha$-relaxation times against $T-T_{c}$, using the critical temperatures of Eq. (2). For all pressures there is a temperature interval, where the data points lie on a straight line in accord with Eq. (7). Deviations from the power-law behavior are visible both at small and large distances from the critical point. The deviations for large $T-T_{c}$ are expected because Eq. (7) is an asymptotic expansion which is only valid, if the reduced distance to $T_{c}$, i.e., $\left(T-T_{c}\right) / T_{c}$, is small. Upper bounds for the validity of Eq. (17) are approximately $0.7(p=0.5), 1.2(p=1)$, and $0.6(p=2)$, which is comparable to experiments [13, 15] and other simulations 20, 21, 22, 23, 24 However, these upper bounds strongly depend on the quantity under consideration. Whereas deviations are very pronounced for the smallest length scale $(q=$ 9.5), Eq. (7) provides a good description at all, except perhaps at the lowest temperatures for the end-to-end distance.

On the other hand, the deviations from the idealised power law at low temperatures could be attributed to the ergodicity restoring jump processes mentioned above. Close to the critical temperature these processes start to contribute significantly to the relaxation dynamics of the system, and therefore the actual relaxation times can be smaller than the predictions of idealised MCT. This behavior has been discussed in experiments, e.g. [13, 15], and simulations, e.g. [19, 22, 23]. Therefore, in practical application of Eq. (77) one faces the problem that its range of validity is limited from below and above, and that it additionally depends on the quantity under consideration.

Furthermore, Eq. (7) implies that in the temperature regime, where the idealised MCT is applicable, the ratio of two different $\alpha$-relaxation times should be independent of temperature. As demonstrated in Fig. 6, this is not the case, even in the regime where the $\beta$-analysis could be done, i.e., for $T-T_{c} \leq 0.07$ [39]. The ratio between different relaxation times can change by almost a factor of two, and the effect is stronger for $q=9.5$ than for 
$q=6.9$ (first minimum and maximum of the static structure factor, respectively). Note that we obtain the same result when applying a different definition of the $\alpha$-relaxation time, which includes the nonergodicity parameter $f_{q}^{\mathrm{sc}}$, i.e., $\phi_{q}^{\mathrm{s}}\left(\tau_{q}\right)=\mathrm{e}^{-1} f_{q}^{\mathrm{sc}}$. However, it is not clear whether this finding is a strong contradiction to MCT, because we have eliminated the dominant temperature dependence, given by Eq. (7), when dividing two relaxation times. Since we are close, but not very close to $T_{c}$, and Eq. (7) is, strictly speaking, only asymptotically valid, one could expect a smooth temperature dependence of prefactors. Such a conclusion can also be drawn from Ref. [16], in which the MCTequations for a model of a colloidal suspension are solved numerically and compared with the asymptotic results. There, it is found that the ratio of two relaxation times becomes constant only very close to the critical point, although Eq. (7) is already observed for larger distance to the critical volume fraction (see Fig. 7 of Ref. [16]). Interestingly, Fig. 6 shows that the ratio is not a monotonic function and exhibits a maximum approximately at the beginning of the temperature interval, in which we can apply ideal MCT to describe the $\alpha$-relaxation time. It seems as if at this temperature a change in the dynamics of the system occurs.

This problem is also reflected in Fig. 7 which shows the results for $\gamma$ when fitting Eq. (77) to the $\alpha$-relaxation time of $\phi_{q}^{\mathrm{s}}(t)$ at $p=1$. The critical temperature was kept fixed $\left(T_{c}=0.45\right)$ in the fits, and the maximum possible number of temperatures was taken into account to determine $\gamma$. It is interesting to note that the $\gamma$-values, determined from Fig. 5 for the different pressures, agree with one another within the error margins so that the following discussion is not specific for $p=1$. Figure 7 shows that the fit procedure yields a decrease of $\gamma$ with decreasing $q$, but the $\gamma$-values are distributed around the result of the $\beta$-relaxation analysis, $\gamma=2.09$ [39]. Alternatively one can keep the exponent $\gamma=2.09$ constant and adjust the critical temperature, $T_{c} \mathrm{~m}$, [39]. Then the critical temperatures for $q \geq 3$ coincide within the error bars with the value obtained from the $\beta$-analysis. However, the diffusion coefficient of a chain yields a $T_{c}$, which is significantly lower. Physically, both types of analysis suggest that going from $T_{c}$ to higher temperatures the melt has a stronger tendency to liquify on short than on the large length scales. Such a behavior is not unique to our polymer model, but It was also found in other simulations 20, 22].

\section{Test of the thermodynamic path independence}

In order to verify the prediction of the thermodynamic path independence, we estimated the density of the melt at the critical temperature $\left(T_{c}=0.45\right)$ of the isobar $p=1$. The density is $\rho=1.042$. Then we performed a number of simulations in the NVT-ensemble at the appropriate isochor (schematically, this is illustrated in Fig. 4), and again calculated various dynamic correlation functions at the simulated temperatures (see Table (1).

The qualitative behaviour of the dynamical correlation functions along this isochor does not differ from the behaviour observed at the various isobars, discussed in the preceding section. As can be seen in Fig. 9 and Fig. 10, which show exemplarily the dynamic correlation of the end-to-end vector orientation, we find again that at lower temperatures a two step relaxation occurs (which cannot be seen on the scale of figure due to the large plateau value), that the relaxation times show a steep increase, and that at least for the lower temperatures the time temperature superposition principle holds. This could have 
been expected, since also earlier simulations of the model in the NVT ensemble had shown such a behaviour [26]. It is interesting to note, however, that, compared to the appropriate isobar, the two step relaxation process can now be observed at higher temperatures already and that for the studied temperatures the $\alpha$-relaxation time is almost one order of magnitude larger.

Once again it is possible to find a temperature region, where the behaviour of the $\alpha$-relaxation times, extracted from the different dynamic correlation functions, can be described by Eq. (7). This is illustrated in Fig. 11, where we show the temperature dependence of various correlation times, as measured in the NVT-ensemble, plotted in such a way that the applicability of the MCT-prediction is clearly demonstrated. Qualitatively, we find the same features, as discussed before for the isobars. There are deviations from linearity at large temperatures, the deviations are more pronounced for the shortest length scales, but still the fits yield very similar values for the critical temperature, which can be combined to:

$$
T_{c}(\rho=1.042)=0.445 \pm 0.010 .
$$

Within our error bars the value coincides with the critical temperature obtained for the isobar $p=1.0$. For the NVT-simulation the error bar is larger, since we were not able to equilibrate the melt as closely to the critical temperature as it was possible in the NpT-simulation. Note that the lowest temperature in Fig. 5b is $T=0.46$, whereas it is $T=0.5$ in Fig. 11. This difference is caused by the larger relaxation time in the NVTensemble (due to higher density/pressure) at a specific temperature in comparison to the NpT-ensemble. Therefore the estimate becomes less accurate, but we can still conclude that the critical temperature of ideal MCT is indeed independent of the thermodynamic path chosen.

Finally, we want to verify whether the exponent $\gamma$ is independent of the thermodynamic path as well. As already discussed in section III, the exponent $\gamma$ shows a pronounced dependence on the dynamic correlation function considered, if one works with the same critical temperature for all quantities and extends the fit interval as much as possible. The same dependence is also found for the isochor, but the results coincide within the error bars with those of the isobars, as Fig. 11 illustrates. Therefore, $\gamma$ is in fact independent of the chosen path, which exemplifies the thermodynamic character of the critical point in mode-coupling theory.

\section{Conclusions}

In this paper we have presented results of a large scale molecular dynamics simulation for a supercooled polymer melt. Our model is a coarse-grained bead-spring model with nonlinear springs connecting monomers along a chain and Lennard-Jones interactions between all monomers. By including competing length scales in the model we prevented the melt from crystallising at lower temperatures.

The present study concentrated on the influence of pressure on the $\alpha$-relaxation behaviour of the melt. Upon cooling we see a steep increase of the $\alpha$-relaxation time, and all dynamic correlation functions show a two-step relaxation. By comparing data 
from different isobars, we found that our system does not only exhibit time-temperaturesuperposition above $T_{c}$, but time-temperature-pressure-superposition as well.

For all pressures investigated it has been possible to locate a temperature interval, where the increase of the $\alpha$-relaxation times could be described by idealised MCT. Therefore we have been able to investigate the dependence of the critical temperature of MCT on pressure and to give a sketch of the critical line in the $(p, T)$-plane. However, whereas the critical temperatures, determined from different quantities probing both small and large length scales of the melt, coincide within the error bars, the approach towards $T_{c}$, i.e., the exponent $\gamma$, is very sensitive to the precise choice of $T_{c}$ in the fit, and depends on the quantity considered. When fixing $T_{c}$, we find that the $\alpha$-relaxation times of $\phi_{q}^{\mathrm{s}}(t)$ for $q$-values distributed around the maximum of the structure factor are compatible with result of the $\beta$-analysis [39]. Deviations occur for much smaller and larger $q$-values. The deviations at large $q$ can be explained by the sensitivity of $\gamma$ on $T_{c}$, since fixing $\gamma$ at the value of the $\beta$-analysis instead of $T_{c}$ yields estimates for $T_{c}$ that are compatible with the result of the $\beta$-analysis [39]. However, such an alternative fit procedure does not remove the discrepancies found on the largest length scale. On these length scales, $\gamma$ is smaller than expected from the $\beta$-analysis. Similar deviations are also observed on smaller length scales, if the critical point is approached very closely. They can be rationalized, within the theoretical framework of MCT, by ergodicity restoring processes, which compete with and finally dominate over the cage effect treated by the idealised theory, if $T \leq T_{c}$. To what extent the predictions of the idealised theory are observable, therefore depends not only on the quantity under consideration, which is also pointed out in recent theoretical work [16, 57], but also on the distance to the critical point. If one is too close, ergodicity restoring processes interfere, and if the temperature is too large, one leaves the asymptotic regime, where the formulas of the idealised MCT are expected to hold.

By performing simulations along an isochor which had the same impact point on the critical line as one of the isobars, we have been able to verify, that within the error margin the critical temperature of MCT is indeed independent of the thermodynamic path one chooses upon cooling. Furthermore, we have shown, that the exponent $\gamma$ does not depend on the thermodynamic path either, within the caveats explained in the last paragraph.

In summary, one can therefore say that the idealised theory is a good starting point for a quantitative description of the dynamics above $T_{c}$, and seems to capture the essential physics, not only for simple liquids, but also for polymer model. Reasons, why this could be the case, are further discussed in Ref. [39].

\section{Acknowledgements}

We would like to thank W. Kob, A. Latz and B. Dünweg for stimulating discussions, and also A. Kopf for supplying his MD-code. Support by the Sonderforschungsbereich SFB 262 , and a generous grant of computer time from the computing center at the university of Mainz and the HLRZ Jülich, are gratefully acknowledged. 


\section{References}

[1] Jäckle J 1986 Rep. Prog. Phys. 49171

[2] Jäckle J 1987 Phil. Mag. B 56113

[3] Zallen R The Physics of Amorphous Solids (New York: Wiley)

[4] McKenna G B 1989 in Comprehensive Polymer Science, Vol. II, edited by C. Booth and C. Price (New York: Pergamon Press) pp. 311-362

[5] Götze W 1993 in Phase Transitions and Relaxations in Systems with Competing Energy Scales, Nato ASI Series C 415, edited by T. Riste and D. Sherrington (Dordrecht: Kluwer Academic Press)

[6] Cohen M H and Turnbull D 1959 J. Chem. Phys. 311164

[7] Cohen M H and Turnbull D 1961 J. Chem. Phys. 34120

[8] Cohen M H and Turnbull D 1970 J. Chem. Phys. 523038

[9] Gibbs J H and Di Marzio E A 1958 J. Chem. Phys. 28373

[10] Gibbs J H and Di Marzio E A 1958 J. Chem. Phys. 28807

[11] Adam G and Gibbs J H 1965 J. Chem. Phys. 43139

[12] Di Marzio E A and Young A J M 1997 J. Res. Natl. Inst. Stand. Technol. 102135

[13] Li G, Du W M, Chen X K, Tao N J and Cummins H Z 1992 Phys. Rev. A 45 3867; Cummins H Z, Du W M, Fuchs M, Götze W, Hildebrand S, Latz A, Li G and Tao N J 1993 Phys. Rev. E 474223

[14] van Megen W and Underwood S M 1993 Phys. Rev. E 47 248; 1994 Phys. Rev. E 49 4206

[15] Cummins H Z, Li G, Du W M and Hernandez J 1994 Physica A 204169

[16] Franosch T, Götze W, Mayr M R and Singh A P 1997 Phys. Rev. E 553183

[17] Tölle A, Schober H, Wuttke J and Fujara F 1997 Phys. Rev. E 56809

[18] Baschnagel J 1994 Phys. Rev. B 49135

[19] Baschnagel J and Fuchs M 1995 J. Phys.: Condens. Matter 76761

[20] Kob W and Andersen H C 1995 Phys. Rev. E 514626

[21] Kob W and Andersen H C 1995 Phys. Rev. E 524134

[22] Kämmerer S, Kob W and Schilling R 1997 Phys. Rev. E 565450

[23] Kämmerer S, Kob W and Schilling R 1998 Phys. Rev. E 582131 
[24] Kämmerer S, Kob W and Schilling R 1998 Phys. Rev. E 582141

[25] Sciortino F, Fabian L, Chen S -H and Tartaglia P 1997 Phys. Rev. E 565397

[26] Bennemann C, Paul W, Binder K and Dünweg B 1998 Phys. Rev. E 57843

[27] Theme Issue on Mode Coupling Theory 1995 edited by S. Yip and P. Nelson, Transport Theory and Statistical Physics 24, No. 6-8

[28] Bengtzelius U, Götze W and Sjölander A 1984 J. Phys. C 1759115

[29] Götze W 1990 in Liquids, Freezing and the Glass Transition, edited by J. P. Hansen, D. Levesque and J. Zinn-Justin (Amsterdam:North-Holland) Part 1 pp. 287-503

[30] Götze W and Sjögren L 1992 Rep. Prog. Phys. 55241

[31] Götze W and Sjögren L 1995 Transport Theory and Statistical Physics 24801

[32] Schilling R 1994 in Disorder Effects on Relaxational Processes, edited by R. Richert and A. Blumen (Berlin: Springer)

[33] Kob W 1997 in Experimental and Theoretical Approaches to Supercooled Liquids: Advances and Novel Application, edited by J. T. Fourkas, D. Kivelson, U. Mohanty and K. A. Nelson (Washington: ACS Books)

[34] Bengtzelius U 1986 Phys. Rev. A 333433

[35] Li G, King H E, Oliver W F, Herbst C A and Cummins H Z 1995 Phys. Rev. Lett. 742280

[36] Tölle A 1997 doctoral thesis (Dortmund)

[37] Tölle A, Schober H, Wuttke J, Randl O G and Fujara F 1998 Phys. Rev. Lett. 80 2374

[38] Angell C A and Sichina W 1976 Ann. NY Acad. Sci. 27953

[39] Bennemann C, Baschnagel J and Paul W, Molecular-Dynamics Simulation of a Glassy Polymer Melt: Incoherent Scattering Function, cond-mat/9809335, submitted to Phys. Rev. E.

[40] Kremer K and Grest G S 1990 J. Chem. Phys. 92 (8) 5057

[41] Dünweg B, Grest G S and Kremer K 1997 conference proceedings of the IMA workshop, May 1996, at the University of Minnesota (Berlin: Springer-Verlag)

[42] Kopf A, Dünweg B and Paul W 1997 J. Chem. Phys. 1076945

[43] Wolfgardt M, Baschnagel J, Paul W and Binder K 1996 Phys. Rev. E 54 (2) 1535

[44] Wolfgardt M and Binder K 1996 Macromol. Theory Simul. 5699

[45] Nosé S 1991 Prog. of Theor. Physics Supplement 1031 
[46] Hoover W G 1985 Phys. Rev. A 311695

[47] Di Tolla D and Ronchetti M 1993 Phys. Rev. E 481726

[48] Bennemann C 1998 doctoral thesis (Mainz)

[49] Schilling R and Scheidsteger T 1997 Phys. Rev. E 562932

[50] Scheidsteger T and Schilling R 1998 Phil. Mag. B 773

[51] Bernu B, Hansen J P, Hiwatari Y and Pastore G 1989 Phys. Rev. A 514891

[52] Roux J N, Barrat J L and Hansen J P 1989 J. Phys. Condens. Matter 17171

[53] Barrat J L and Latz A 1990 J. Phys. Condens. Matter 24289

[54] Nauroth M and Kob W 1997 Phys. Rev. E 55657

[55] Lunkenheimer P, Pimenov A, Dressel M, Schiener B, Schneider U and Loidl A 1997 Prog. Theo. Phys. Suppl. 126123

[56] Bennemann C, Paul W, Baschnagel J and Binder K Molecular-Dynamics Simulation of a Glassy Polymer Melt: Rouse Modes and Mean-Square Displacements, preprint

[57] Fuchs M, Götze W and Mayr M R submitted to Phys. Rev. E 
Table 1: The table shows, at which temperatures and densities or pressures simulations were performed.

\begin{tabular}{|l|l|}
\hline ensemble & simulated temperatures \\
\hline \hline isochor $(\rho=1.042)$ & $0.5,0.52,0.55,0.58,0.6,0.65,0.7,0.8,0.9,1.0,2.0$ \\
\hline isobar $(p=0.5)$ & $0.45,0.48,0.5,0.52,0.55,0.6,0.7,1.0$ \\
\hline isobar $(p=1.0)$ & $0.46,0.47,0.48,0.49,0.5,0.52,0.55,0.6,0.65,0.7,1.0,2.0,4.0$ \\
\hline isobar $(p=2.0)$ & $0.52,0.55,0.57,0.6,0.7,0.8,0.9,1.0,2.0$ \\
\hline
\end{tabular}

Table 2: Critical temperatures and densities and soft sphere scaling variable at the critical point

\begin{tabular}{|l|l|l|l|}
\hline$p$ & $T_{c}$ & $\rho_{c}$ & $\rho_{c} T_{c}^{-1 / 4}$ \\
\hline 0.5 & $0.425 \pm 0.010$ & $1.035 \pm 0.01$ & $1.28 \pm 0.02$ \\
\hline 1.0 & $0.450 \pm 0.005$ & $1.042 \pm 0.01$ & $1.27 \pm 0.02$ \\
\hline 2.0 & $0.490 \pm 0.010$ & $1.054 \pm 0.01$ & $1.26 \pm 0.02$ \\
\hline
\end{tabular}




\section{Figure Captions}
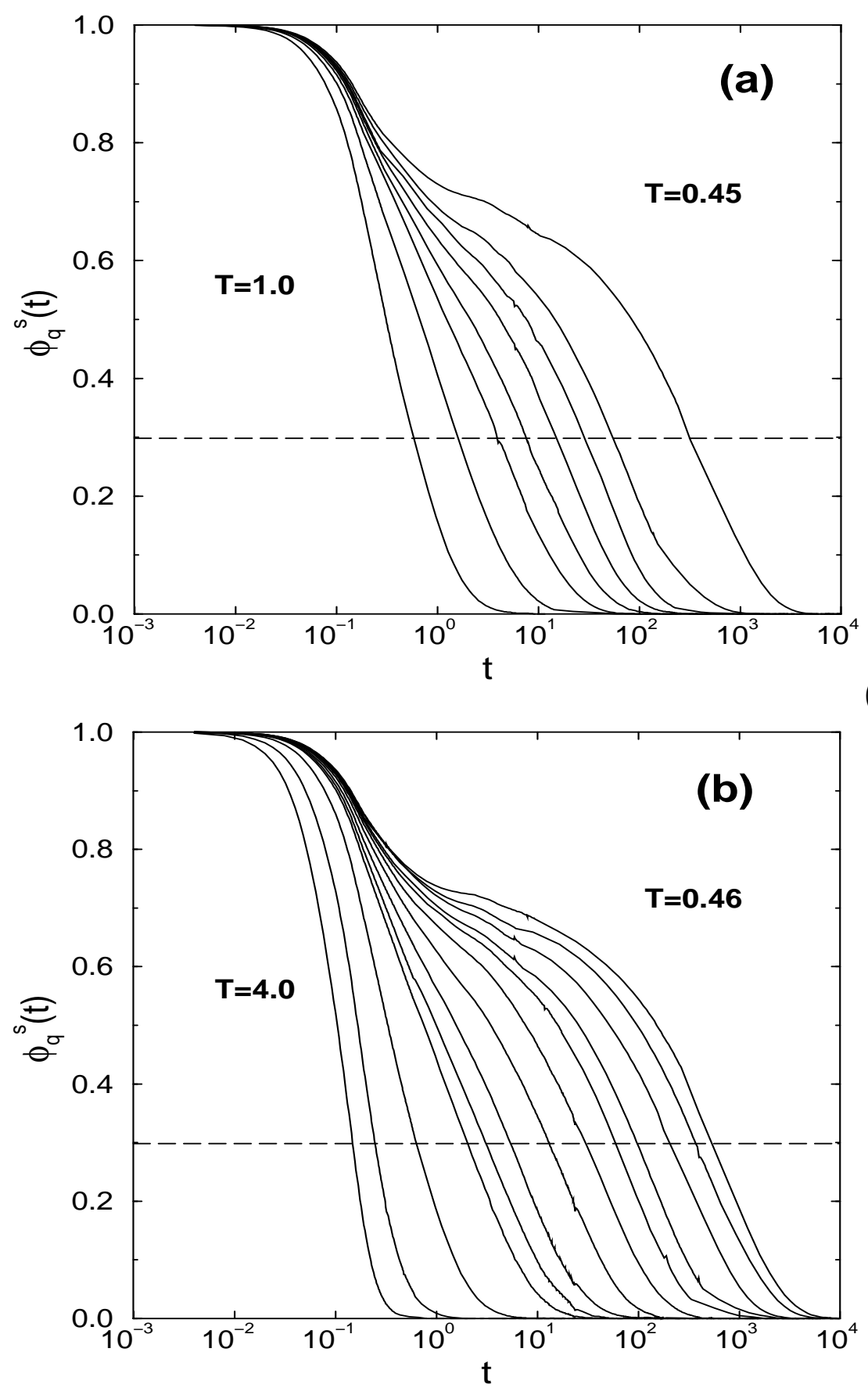

(a)

(b) 


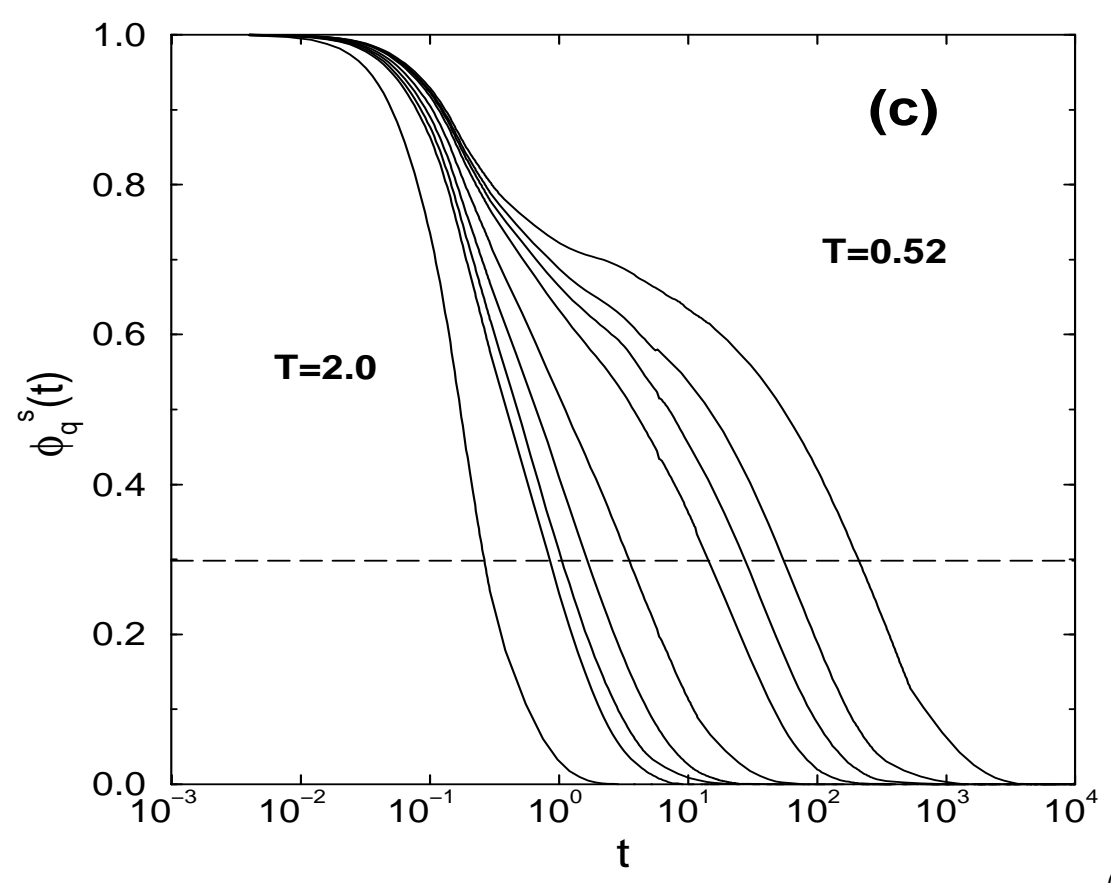

(c)

Figure 1: Intermediate dynamic structure factors at the first maximum of the static structure factor $(q=6.9)$ 39 measured along the isobars $p=0.5(\mathrm{a}), p=1.0$ (b) and $p=2.0$ (c). The broken line shows the value, which we used to define the $\alpha$-relaxation time scale. From right to left, the temperatures decrease, as specified in Table 1 . 


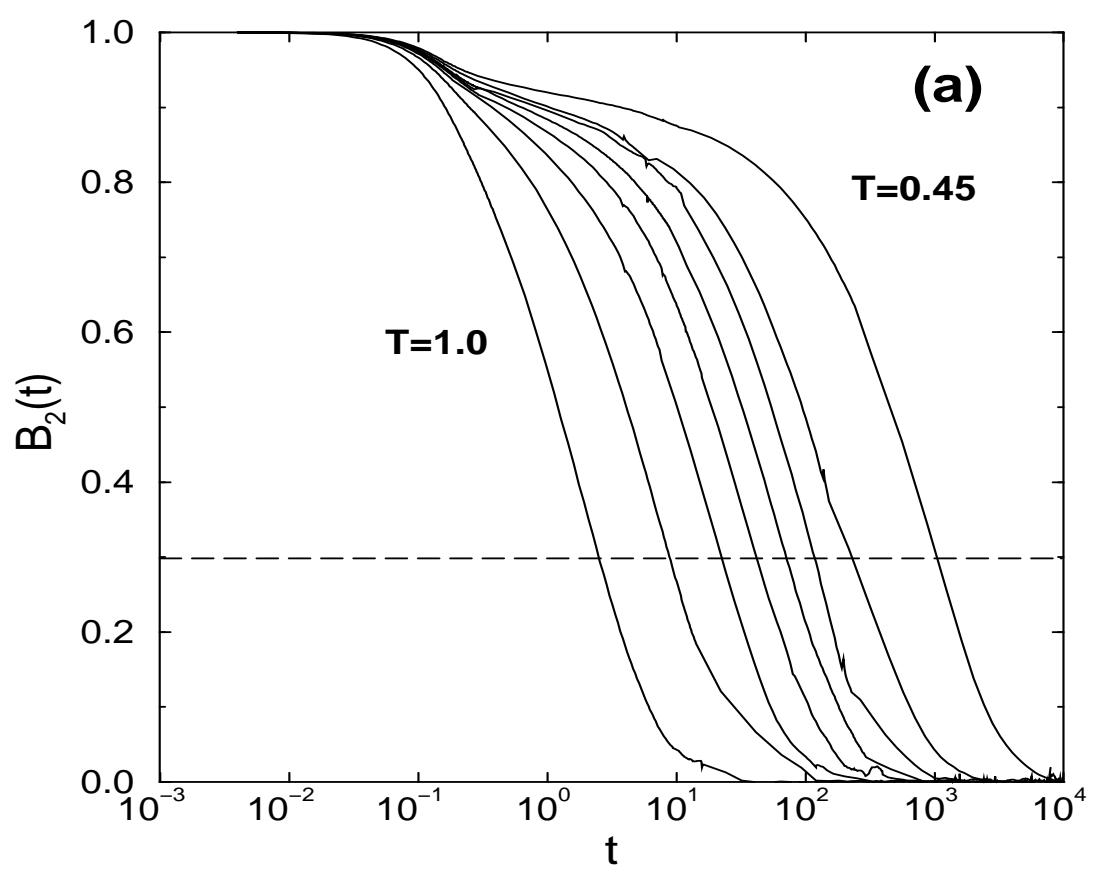

(a)

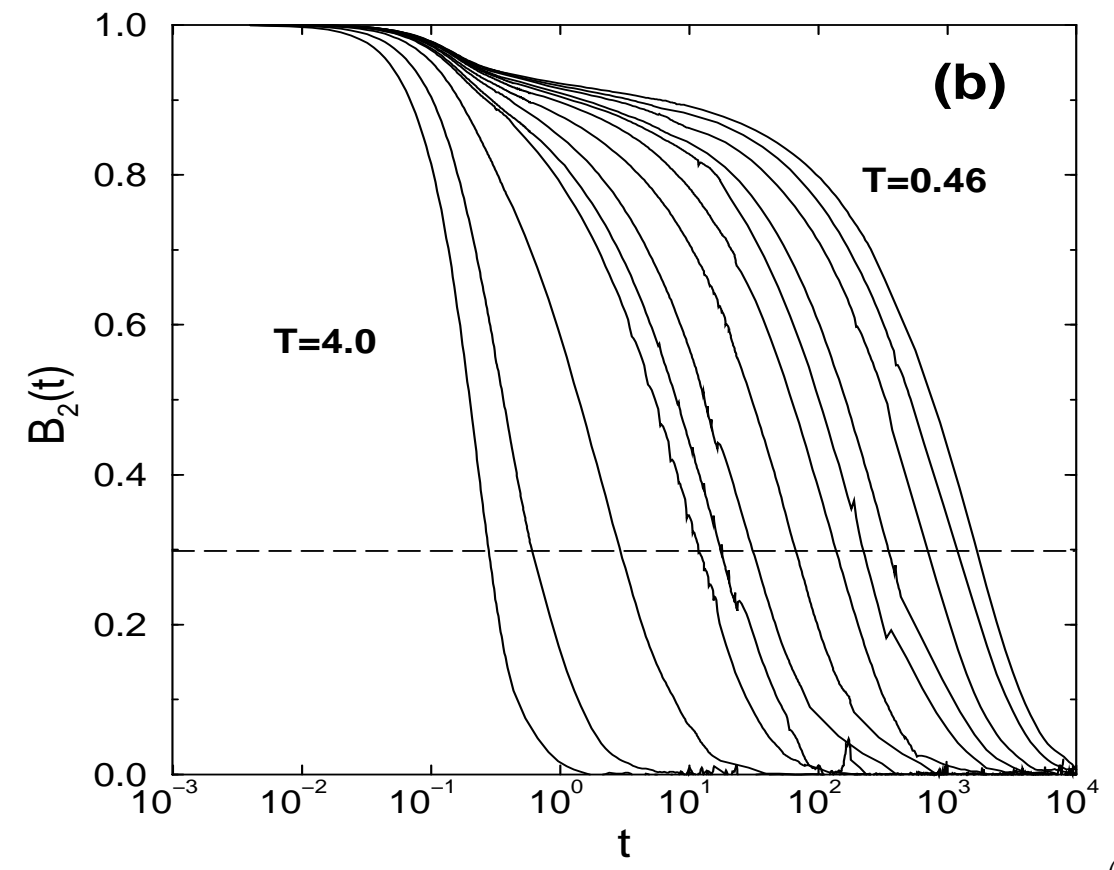

(b) 


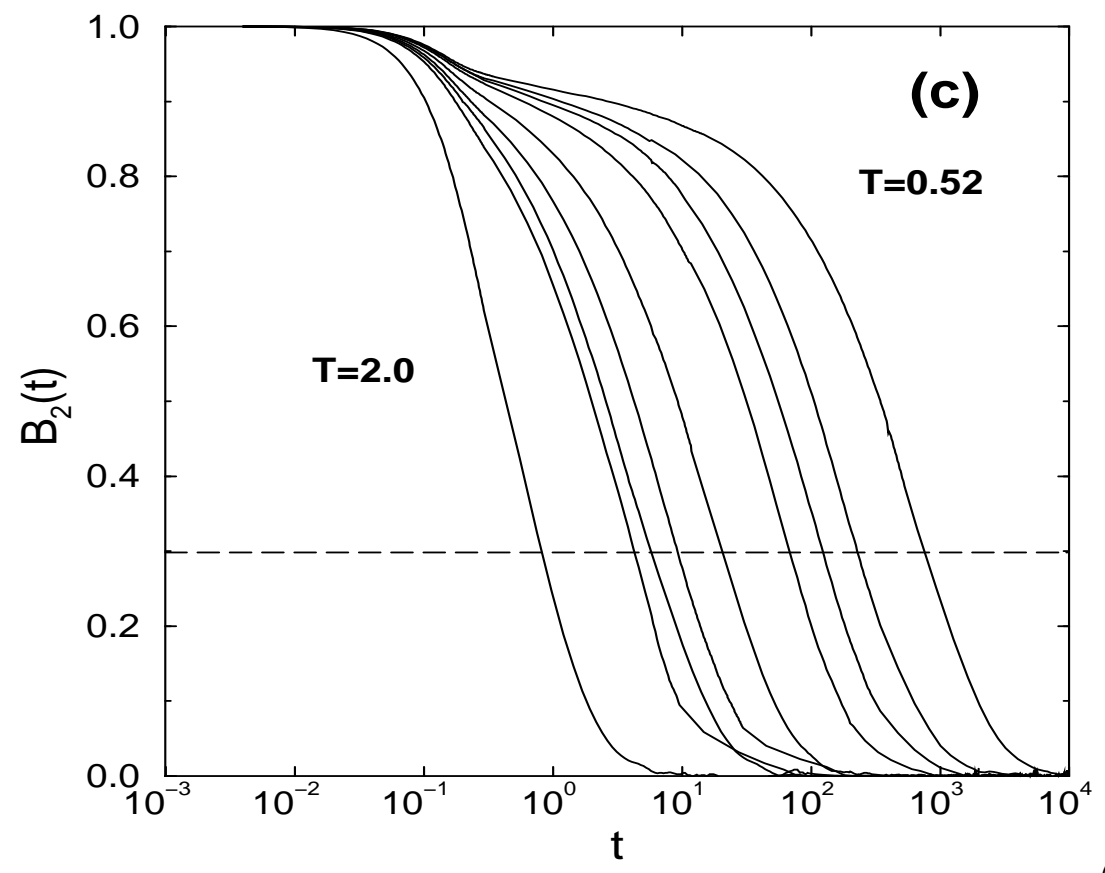

(c)

Figure 2: Dynamic correlation functions of the orientation of the bond vectors [second Legendre polynomial, see Eq. (5)]. Different pressures are shown: $p=0.5$ (a), $p=1.0$ (b) and $p=2.0$ (c). From right to left, the temperatures decrease, as specified in Table 1 . 


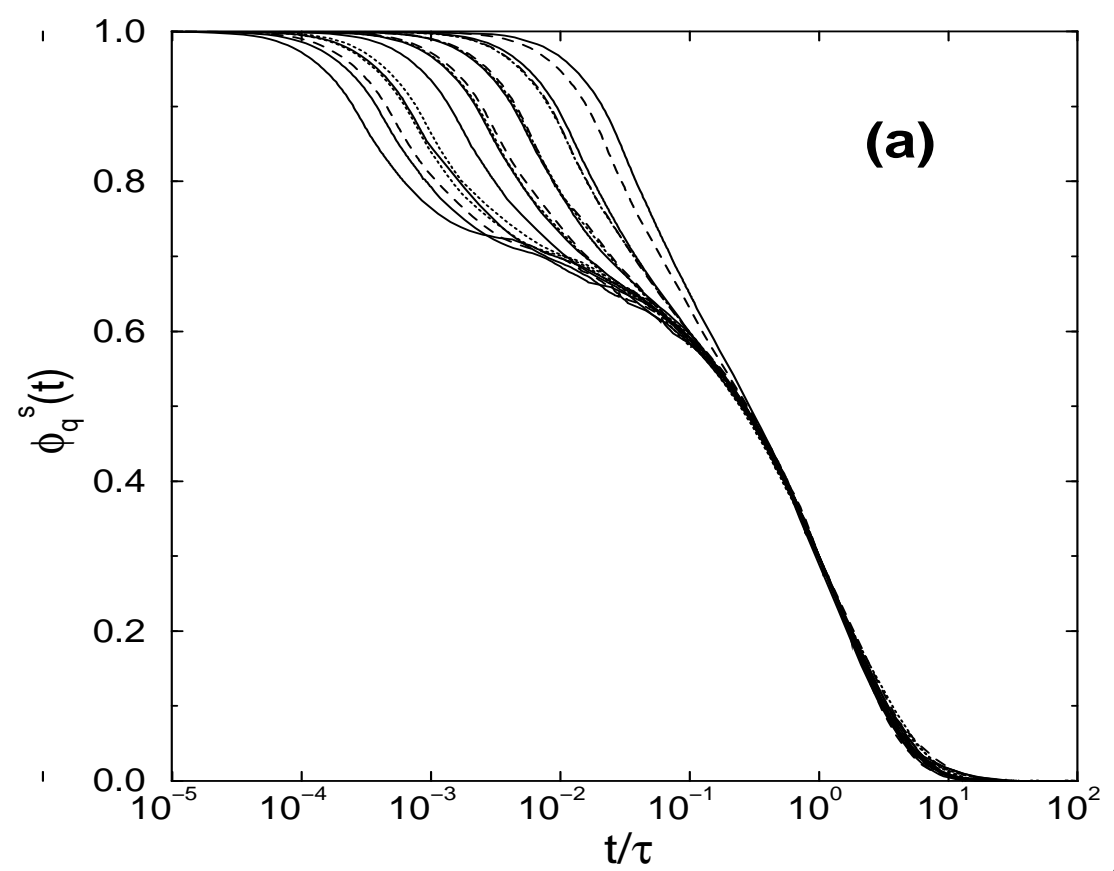

(a)

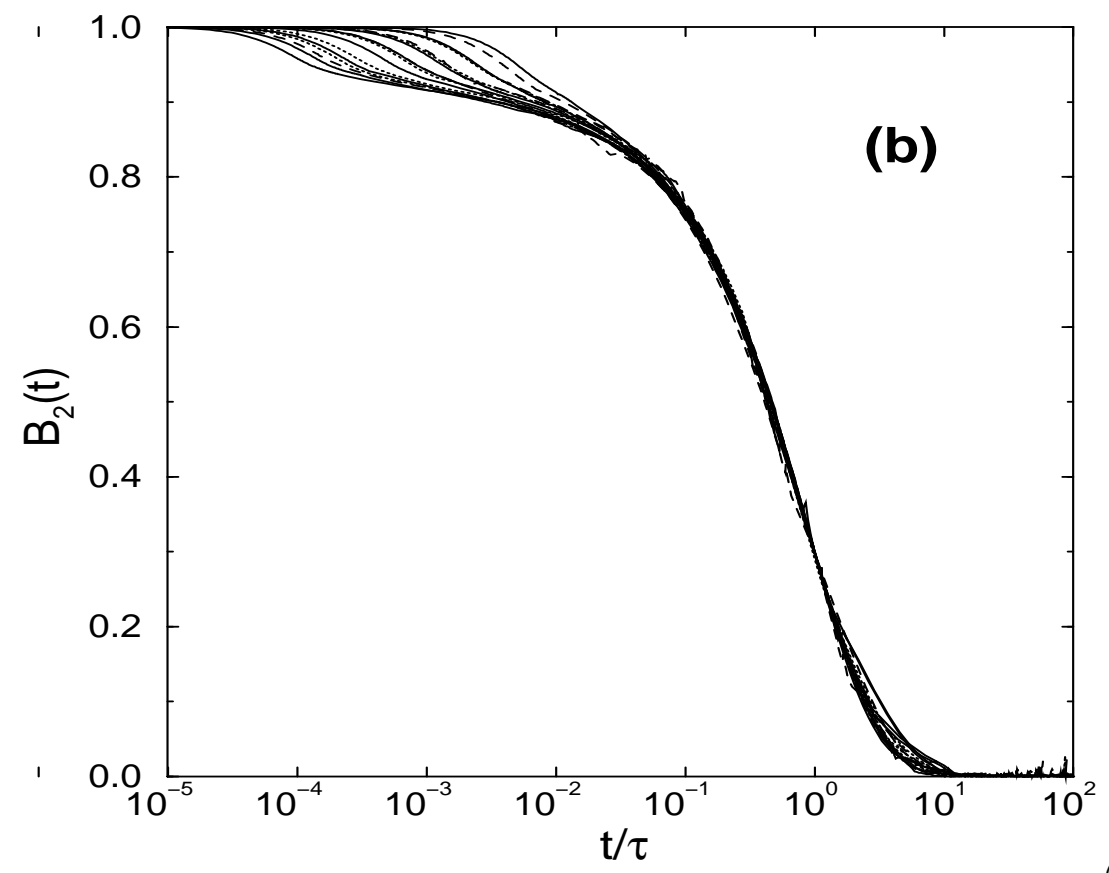

(b) 


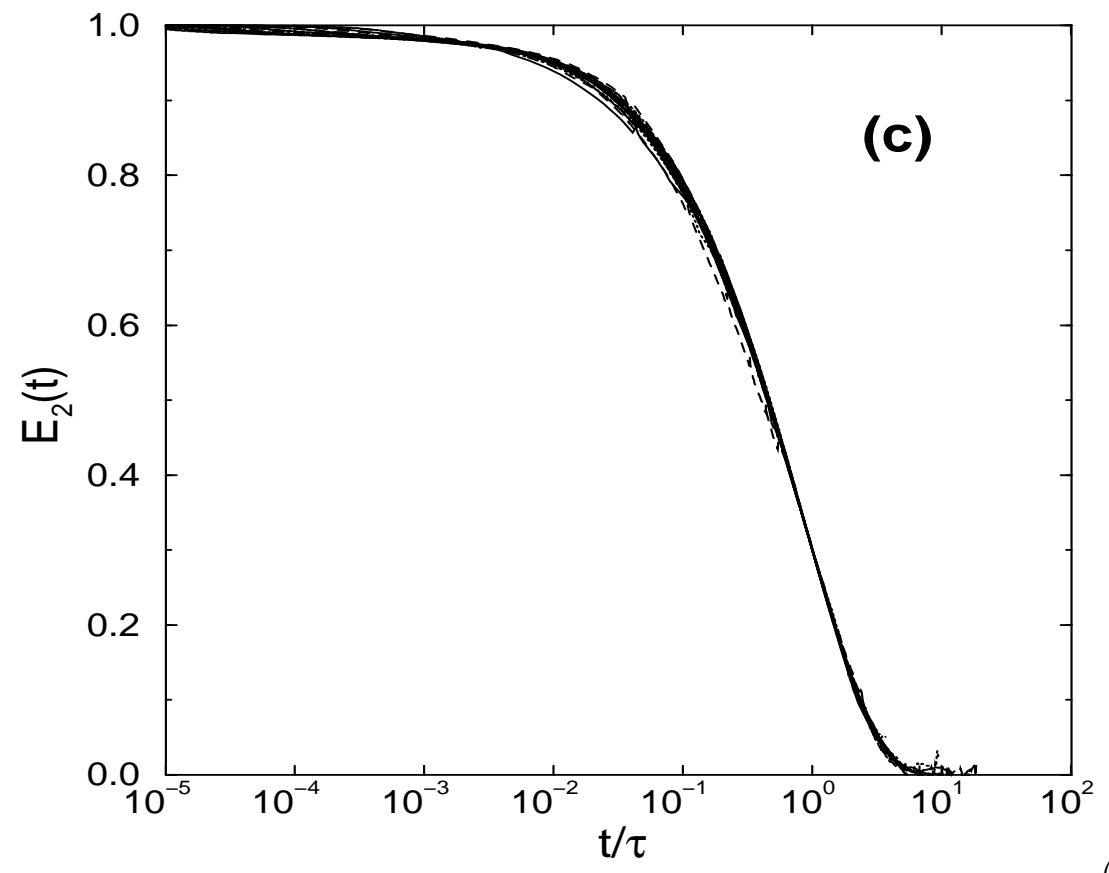

(c)

Figure 3: Figure. 3a is a compilation of the results from Figs. 1a-c, but with times scaled by the $\alpha$-relaxation time scale for selected temperatures close to the critical temperature at the respective pressure: $T=0.45,0.48,0.5,0.52,0.55$ for $p=0.5$ (dashed lines), $T=$ $0.46,0.47,0.48,0.5,0.52$ for $p=1$ (solid lines) and $T=0.52,0.55,0.57,0.6$ for $p=2$ (dotted lines). In the $\alpha$-regime the curves for different temperatures and pressures all collapse on a single master curve, demonstrating time-temperature-pressure superposition. Figures $3 \mathrm{~b}$ and $3 \mathrm{c}$ show the same behavior for the orientational correlation functions $B_{2}(t)$ and $E_{2}(t)$ (second Legendre polynomial) of the bonds and the end-to-end vector. Note that the plateau for $E_{2}(t)$ is so close to 1 that the first step cannot be seen on the scale of the figure. 


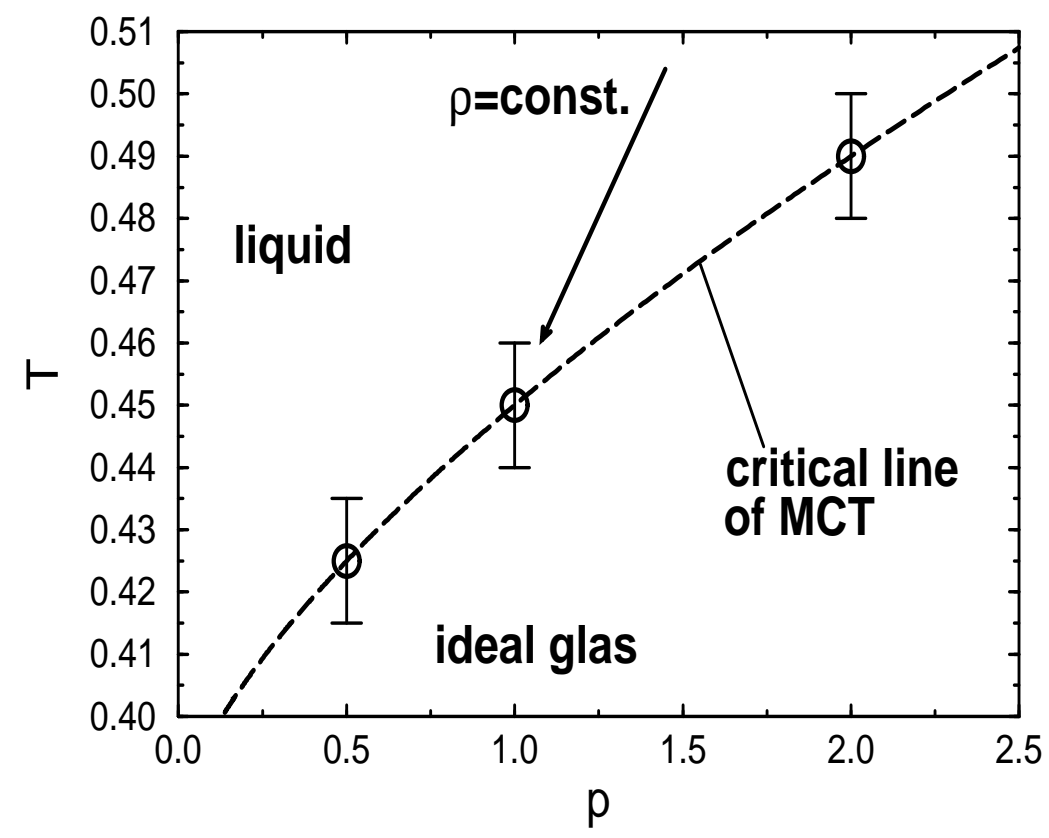

Figure 4: Mode Coupling critical temperatures at different pressures. The critical temperatures represent averages which are derived by fitting Eq. (7) to all relaxation times shown in Fig. 5. The broken line is an illustration of the critical line of MCT (guide to the eye only), while the arrow symbolises a thermodynamic path at constant density. 

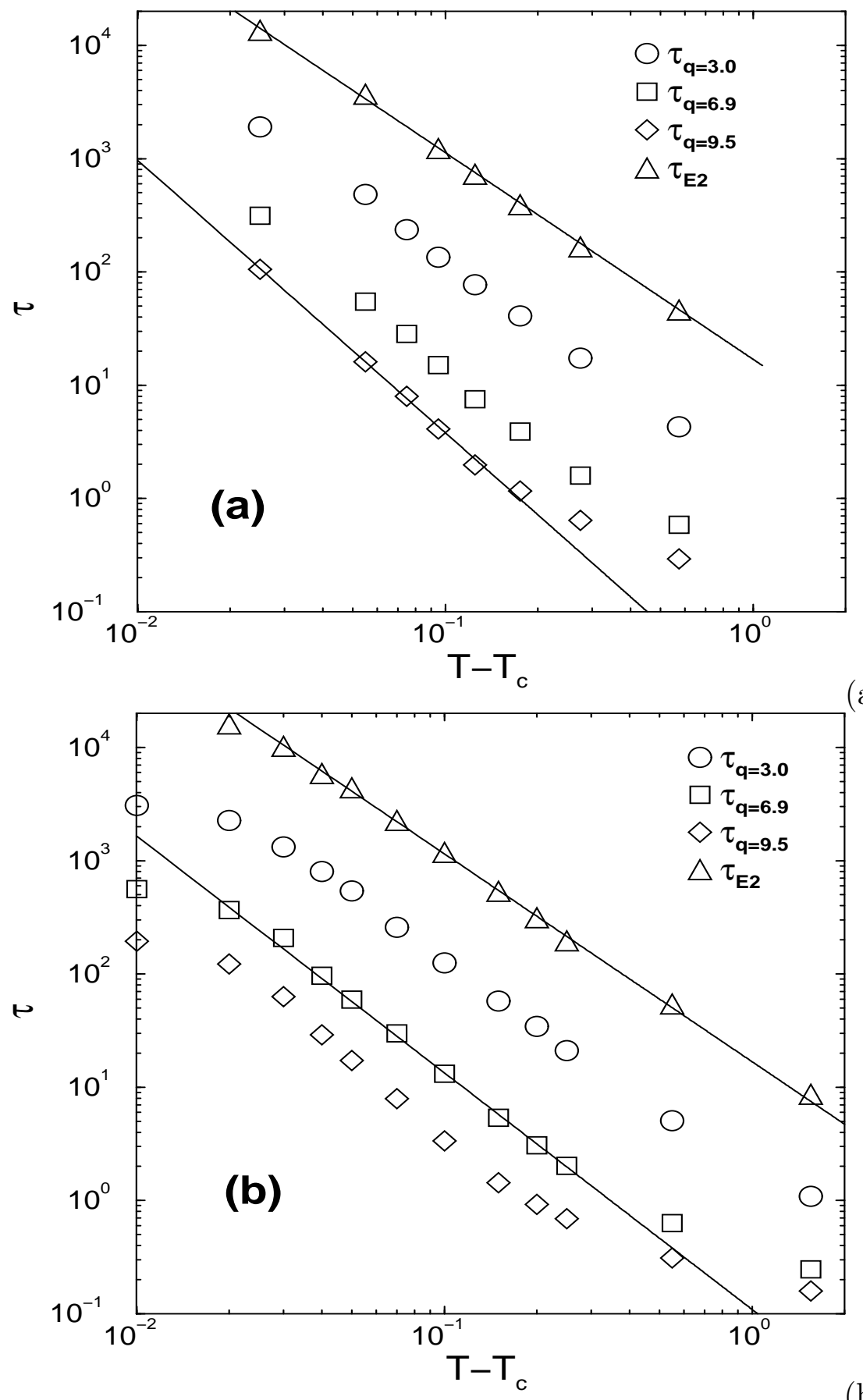

(a) 


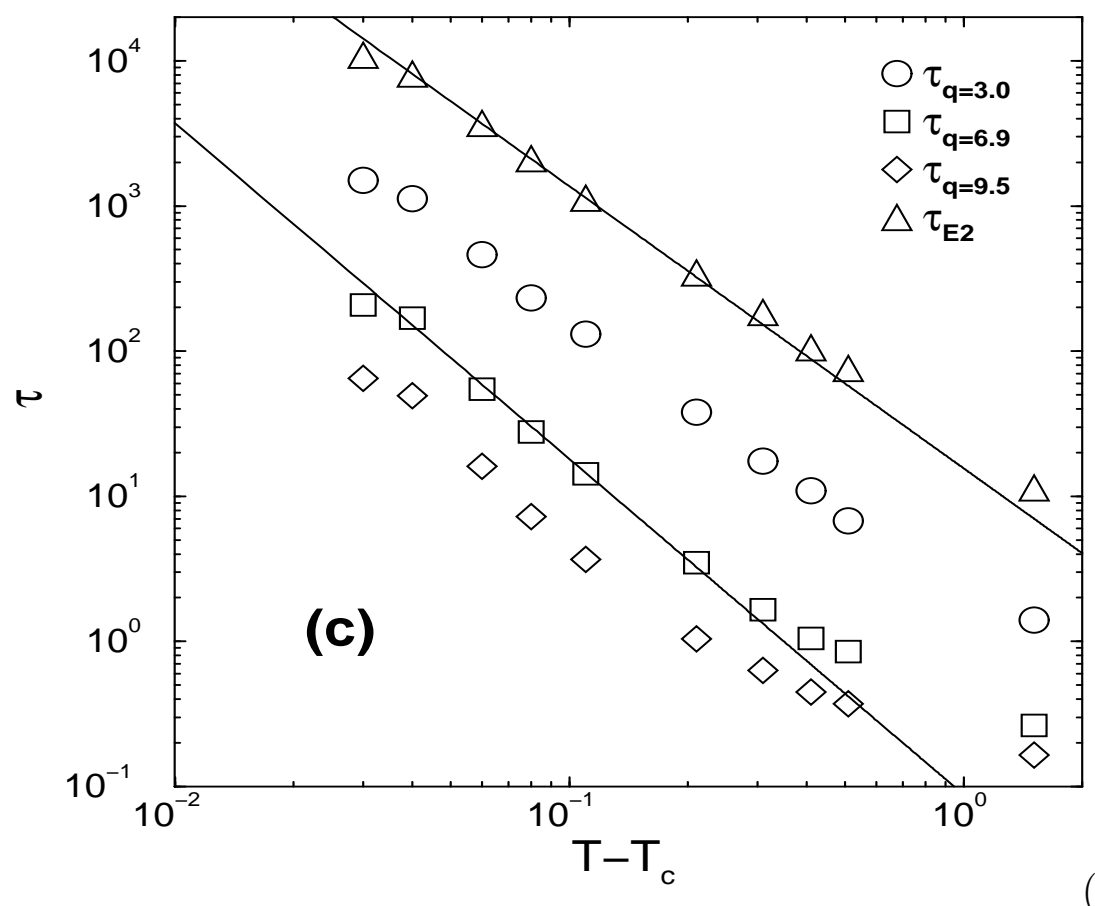

(c)

Figure 5: Temperature behaviour of different relaxation times, measured along the isobars $p=0.5$ (a), $p=1.0$ (b) and $p=2.0$ (c). In the plots $\tau_{q}$ and $\tau_{E_{2}}$ are the $\alpha$-relaxation times of the incoherent dynamic structure factor at different wave numbers and the dynamic orientational correlation of the end-to-end vector (second Legendre polynomial), respectively. The values of $T_{c}$ are taken from Eq. (2). The solid lines are power-law fits, including the largest possible number of temperatures. For $p=1$, the fit for $q=6.9$ uses $\gamma=2.09$, i.e., the $\gamma$-value resulting from an analysis of the $\beta$-relaxation [39]. 


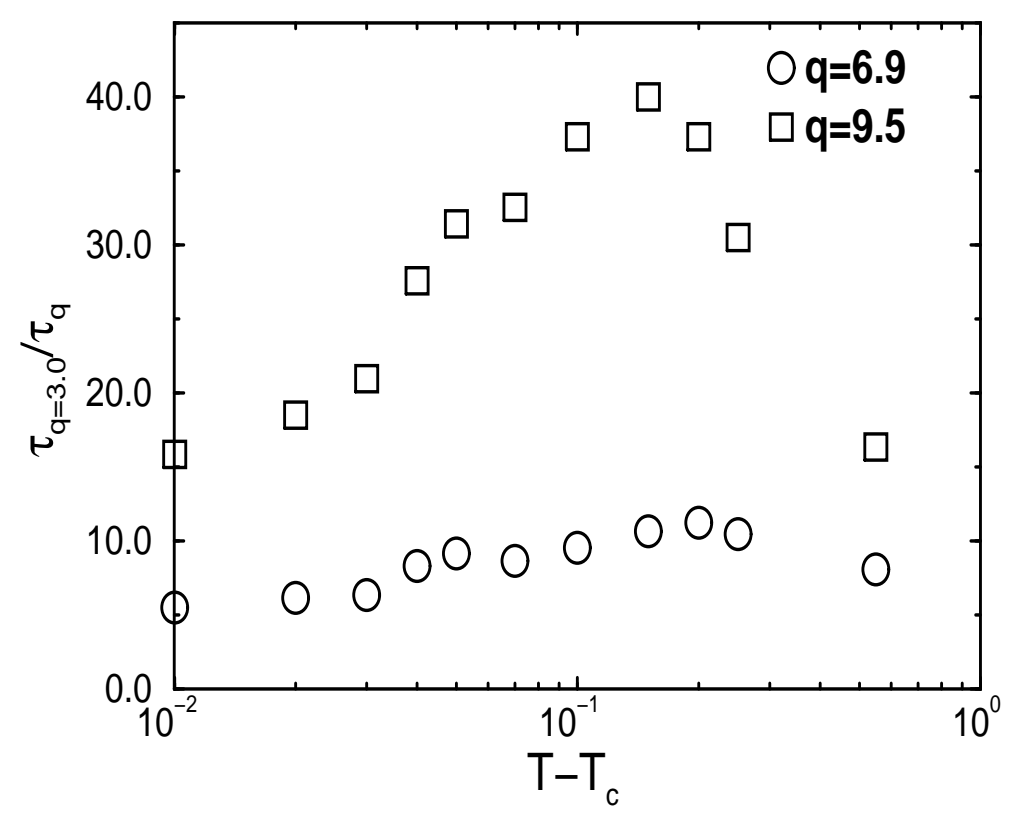

Figure 6: Ratio of different $\alpha$-relaxation times as measured along the isobar $p=1.0$. As can be seen, even close to the critical temperature $\left(T_{c}=0.45\right)$ the ratio changes by almost a factor of two.

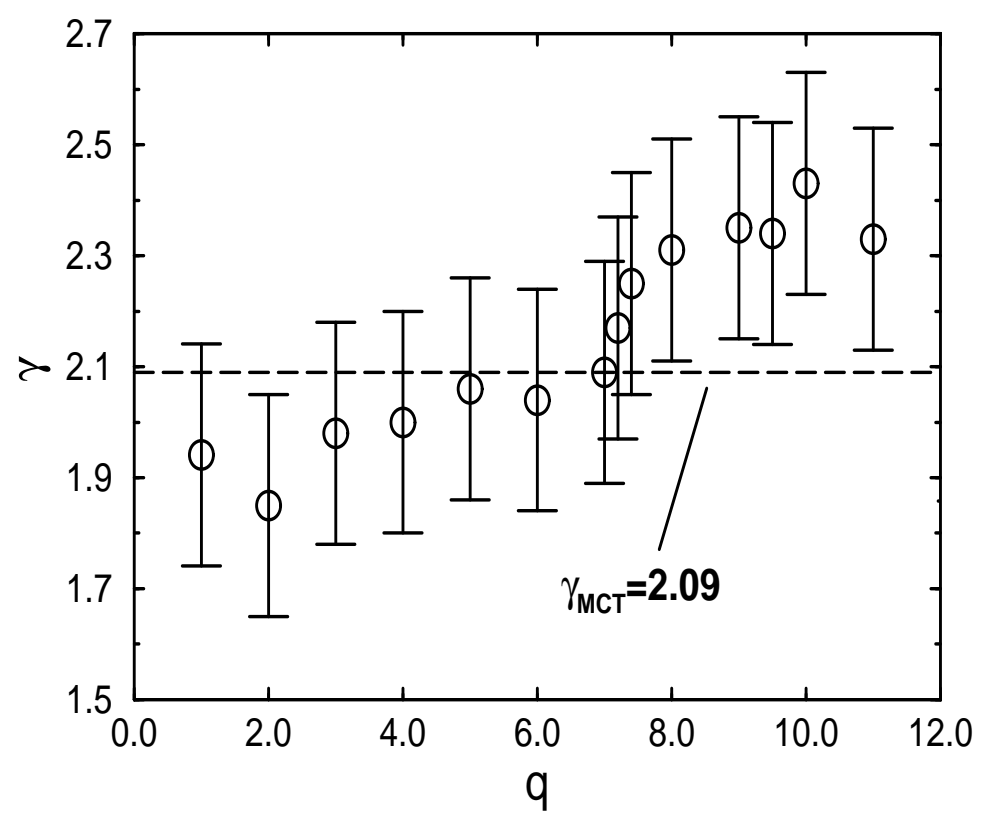

Figure 7: Variation of $\gamma(p=1.0)$ with the magnitude $q$ of the wave-vector when fitting the $\alpha$-relaxation time of $\phi_{q}^{\mathrm{s}}(t)$ by Eq. (7) while keeping the critical temperature fixed $\left(T_{c}=0.45\right)$. 


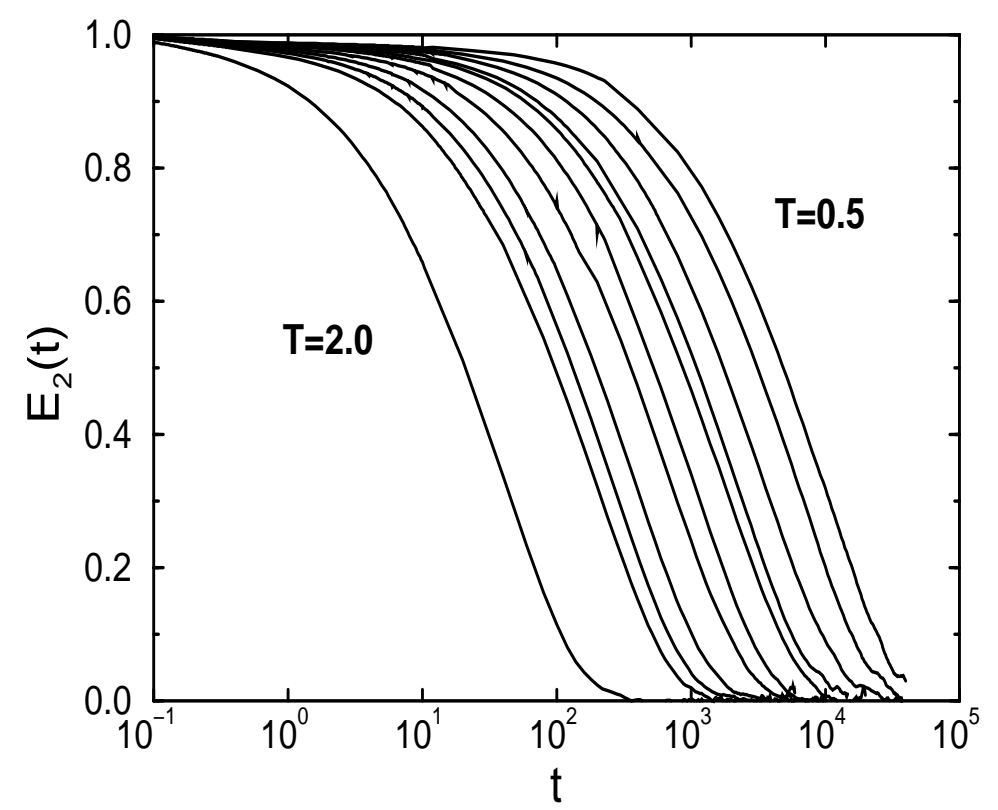

Figure 8: Dynamic correlation of the end-to-end vector orientation (second Legendre polynomial), as measured at constant density along the thermodynamic path $\rho=1.042$. Temperature decreases from right to left, as specified in Table 1 .

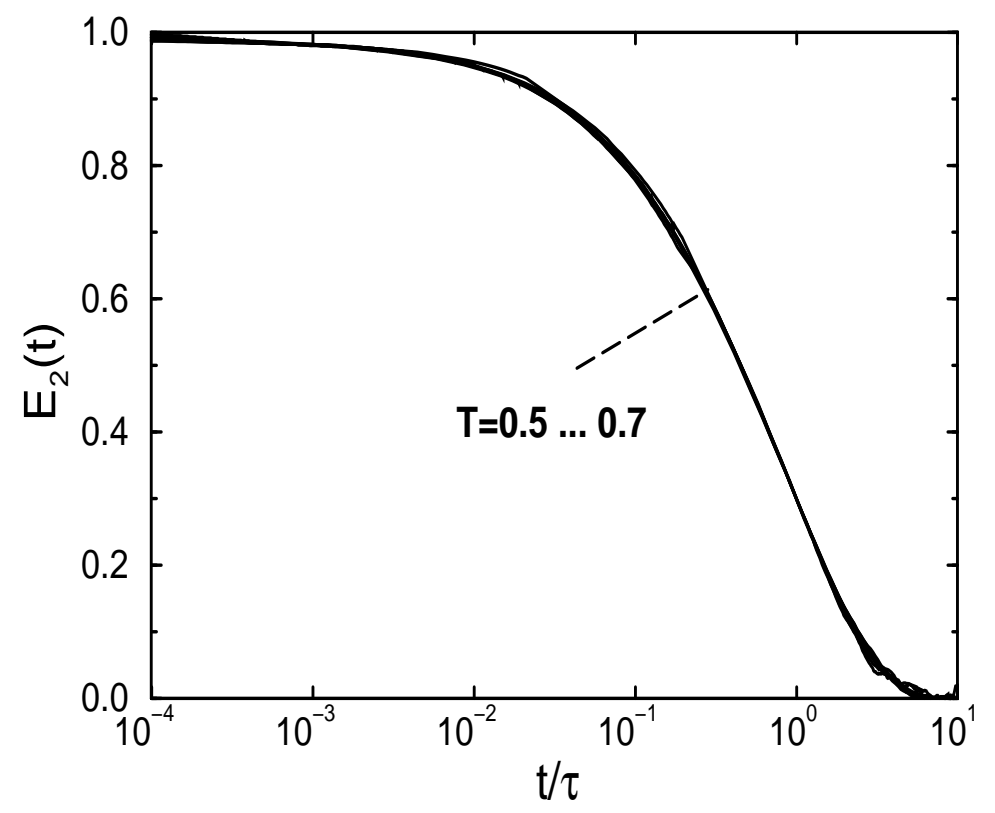

Figure 9: $\alpha$-scaling plot of the end-to-end vector correlation function for temperatures ranging from $T=0.5$ to $T=0.7$ (see Table 1 for details). The simulation data are for the same isochor as in Fig. 8, and the relaxation time was determined by Eq. (6). 


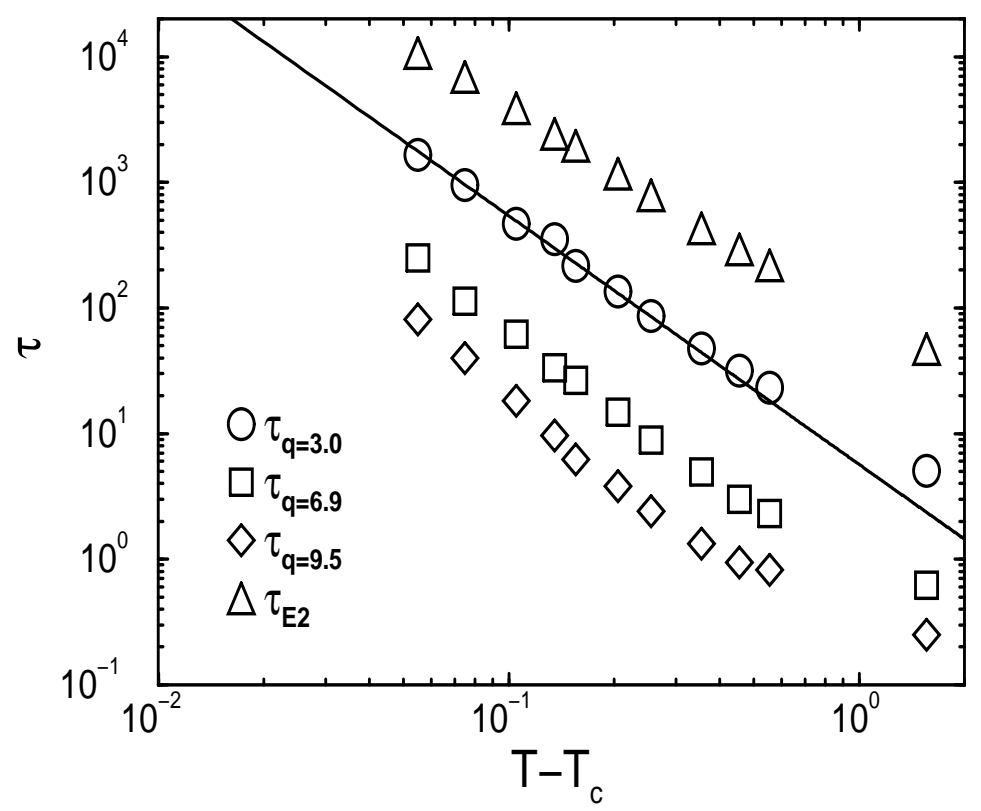

Figure 10: Temperature behaviour of different $\alpha$-relaxation times, as measured in the NVT-ensemble. The solid line represents a fit with Eq. (7).

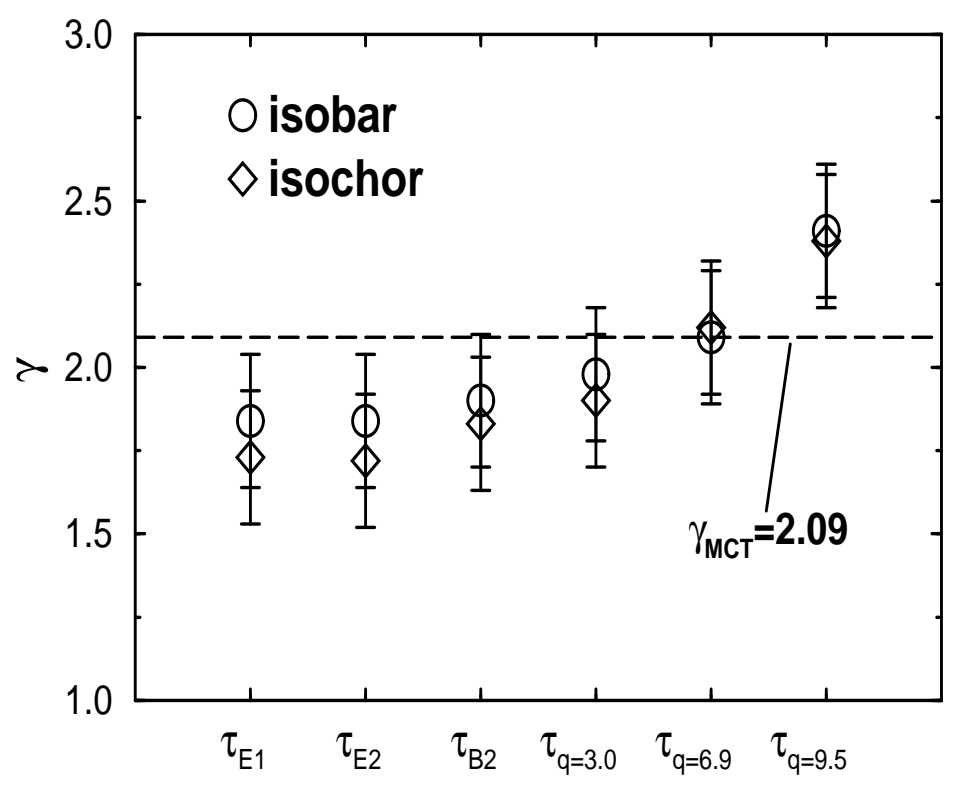

Figure 11: Values of $\gamma$, determined from the temperature dependence of various correlation times, for two different thermodynamic paths, which yield the same critical temperature. On the abscissa, the relaxation times are quoted, from which $\gamma$ was determined. For both the isobaric and the isochor path the error margins are about $10 \%$, which is rather large, since $\gamma$ is very sensitive to a variation of the critical temperature. Within the error bars, $\gamma$ does not depend on the thermodynamic path. 